\title{
Comparison of different annotation tools for characterization of the complete chloroplast genome of Corylus avellana cv Tombul
}

\author{
Kadriye Kahraman ${ }^{1,2}$ and Stuart James Lucas ${ }^{2 *}$
}

\begin{abstract}
Background: Several bioinformatics tools have been designed for assembly and annotation of chloroplast (cp) genomes, making it difficult to decide which is most useful and applicable to a specific case. The increasing number of plant genomes provide an opportunity to accurately obtain cp genomes from whole genome shotgun (WGS) sequences. Due to the limited genetic information available for European hazelnut (Corylus avellana L.) and as part of a genome sequencing project, we analyzed the complete chloroplast genome of the cultivar 'Tombul' with multiple annotation tools.
\end{abstract}

Results: Three different annotation strategies were tested, and the complete cp genome of $C$. avellana cv Tombul was constructed, which was 161,667 bp in length, and had a typical quadripartite structure. A large single copy (LSC) region of 90,198 bp and a small single copy (SSC) region of 18,733 bp were separated by a pair of inverted repeat (IR) regions of $26,368 \mathrm{bp}$. In total, 125 predicted functional genes were annotated, including 76 proteincoding, 25 tRNA, and 4 rRNA unique genes. Comparative genomics indicated that the cp genome sequences were relatively highly conserved in species belonging to the same order. However, there were still some variations, especially in intergenic regions, that could be used as molecular markers for analyses of phylogeny and plant identification. Simple sequence repeat (SSR) analysis showed that there were 83 SSRs in the cp genome of cV Tombul. Phylogenetic analysis suggested that $C$. avellana cv Tombul had a close affinity to the sister group of $C$. fargesii and C. chinensis, and then a closer evolutionary relationship with Betulaceae family than other species of Fagales.

Conclusion: In this study, the complete cp genome of Corylus avellana cv Tombul, the most widely cultivated variety in Turkey, was obtained and annotated, and additionally phylogenetic relationships were predicted among Fagales species. Our results suggest a very accurate assembly of chloroplast genome from next generation whole genome shotgun (WGS) sequences. Enhancement of taxon sampling in Corylus species provide genomic insights into phylogenetic analyses. The nucleotide sequences of $\mathrm{cv}$ Tombul $\mathrm{cp}$ genomes can provide comprehensive genetic insight into the evolution of genus Corylus.

Keywords: Corylus avellana, Tombul cultivar, Hazelnut, Chloroplast genome, Phylogeny

\footnotetext{
*Correspondence: slucas@sabanciuniv.edu

${ }^{2}$ Sabanci University Nanotechnology Research and Application Centre

(SUNUM), Sabanci University, 34956 Istanbul, Turkey

Full list of author information is available at the end of the article
}

(c) The Author(s). 2019 Open Access This article is distributed under the terms of the Creative Commons Attribution 4.0 International License (http://creativecommons.org/licenses/by/4.0/), which permits unrestricted use, distribution, and reproduction in any medium, provided you give appropriate credit to the original author(s) and the source, provide a link to the Creative Commons license, and indicate if changes were made. The Creative Commons Public Domain Dedication waiver (http://creativecommons.org/publicdomain/zero/1.0/) applies to the data made available in this article, unless otherwise stated. 


\section{Background}

European hazel (Corylus avellana L.) is a crop tree of worldwide agronomic importance, which has been cultivated for human consumption for thousands of years with a large geographic distribution [1]. Hazelnuts are high in unsaturated fats and contain many essential and minerals, and thereby $C$. avellana occupies an important place in human nutrition [2]. Broad usage of C. avellana, such as adding flavor and texture to dairy, bakery, confectionary and chocolate products, indicate its value to the food industry. Even though it has a significant place in agriculture, a limited number of studies exists about $C$. avellana at the molecular level. Currently the only available genome sequences for C. avellana is a draft genome for the American cultivar 'Jefferson' [3]. In this study, we report the chloroplast genome sequences of Tombul cultivar, the most widely grown Turkish variety, from next generation whole genome shotgun sequences.

The chloroplast (cp) is the main site of photosynthesis and contains enzymatic mechanisms for carbohydrate biosynthesis. The cp genomes of plants are highly conserved in terms of gene size, content and organization, and have a simple circular, quadripartite structure, including two copies of an inverted repeat (IR) that separate the large and small single copy regions (LSC and $\mathrm{SSC}$ ). Because of its conserved nature, the cp genome contributes to plant systematics and evolutionary studies [4-6]. In addition, due to their small genome size, it is much easier to compare cp genomes than the whole genomic data for genomic comparative analysis. Early on chloroplast DNA (cpDNA) fragments are often used as 'DNA barcodes' in inter-species phylogenetic analysis due to their universal presence and abundance in plant cells. However, Yang et al. [7] indicated that the cpDNA fragments most commonly used in phylogenetic analysis such as $m a t K, r b c L$ and $t r n H-p s b A$, have little sequence divergence in genus Corylus, thus it is hard to precisely resolve phylogenetic relationships within the genus using these fragments. Especially in the phylogeny of land plants, studies demonstrated that complete chloroplast genomes provide more reliable information than cpDNA barcode sequences, and eliminate problems associated with barcoding, such as primer design and amplification [8-12]. The complete cp genomes are useful and costeffective for resolving phylogenetic relationship at both high and low taxonomic levels because they contain both conserved and variable protein-coding genes; also, compared to the nuclear genome $\mathrm{cp}$ genomes exhibit a slower evolutionary rate and mostly uniparental inheritance [13-19]. Limited sequence variation has led to the use of cp genomes mostly in studies at the interspecific and interfamilial levels $[13,14,20,21]$. In addition, $c p$ genomes provide deeper information for phylogeny reconstruction of Corylus species in comparison with previous studies that relied on molecular markers, including RAPD [22], SSR [23, 24], SRAP [25], ISSR [26, 27], AFLP [28], and DNA fragments such as ITS regions and cpDNA fragments [28-30]. The whole cp genome is also useful for identification of plant varieties by allowing selection of highly variable non-genic markers for DNA barcoding [31, 32].

Barker et al. [33] indicate that next generation whole genome shotgun (WGS) sequences from plants typically contain $5 \%$ or more reads derived from the chloroplast. Thus, the sequenced genome data of plant species can be used to obtain cp genomes without prior isolation of cpDNA. Due to the development of next generation sequencing technology, an increasing number of WGS datasets are available for $\mathrm{cp}$ genome assembly. Wang et al. [34] revealed the complete cp genomes of Fagopyrum dibotrys from high-throughput sequencing datasets, and obtained reliable chloroplast genomes. Osuna-Mascaró et al. [35] also retrieved the cp genome of Erysimum (Brassicaceae) species from a genomic library, and achieved similar cp genomes in terms of overall size, structure and composition. Besides de novo assembly of complete chloroplast genome, alignment-based methods can also be used to obtain cp assemblies from WGS reads by mapping them onto a reference cp genome [36]. However, this latter method relies on the availability of a high quality cp genome from a related species.

Herein, we present the complete cp genome of Corylus avellana cv Tombul. The aim of the study was to compare different available annotation tools, develop an optimized pipeline for $\mathrm{cp}$ assembly and annotation form WGS sequences, and examine the cp genome structure, gene content and gene order of Turkish hazelnut. Although there is a chloroplast genome for C. avellana in NCBI (KX822768), there is no detailed information about the construction of this genome or which variety of hazelnut it originates from. Therefore, we chose to generate a new annotation for one of the most commercially important Turkish hazelnut cultivars, 'Tombul'. Moreover, simple sequence repeats (SSRs) are investigated in cv Tombul cp genome, and phylogenetic relationships are predicted among the Fagales, including genera Betulaceae, Fagaceae and Juglandaceae.

\section{Results}

Size, gene content, order and organization of the hazelnut

Initial assembly using the NOVOplasty assembler with raw C.avellana cv 'Tombul' WGS sequences produced a single 200,017 bp contig [37]. The length of this contig was significantly longer than the $C$. avellana cp genome previously published in GenBank (Accession no: KX822768). Therefore, the raw contig was aligned to the KX822768 cp genome, and it was observed that the last 
part, starting from $161,667 \mathrm{bp}$, consisted of repeats of sequences from the rest of the Tombul chloroplast genome. To demonstrate whether the extra part, located after 161,667 bp, was genuine or not, Nanopore sequencing reads belonging to $\mathrm{cv}$ Tombul were also aligned to the contig. Although a subset of reads matched these additional parts in two segments, the mapped read depth of the these segments was approximately half of that of the rest of the cp genome. Moreover, BLAST alignment found that the additional part was $100 \%$ identical to two regions in the first $161 \mathrm{~kb}$ of the $\mathrm{cv}$ Tombul cp genome [38]. These observations suggested that the extra $39 \mathrm{~kb}$ in our initial contig was an artefact of the NOVOplasty assembly algorithm, where the duplicated segments were incorporated twice, perhaps due to sequence variation at their boundaries.

In addition, we examined whether a single circular cp genome could be retrieved using a standard whole genome assembly algorithm, rather than one specific to the chloroplast. For this test, trimmed WGS sequences were assembled using ABySS assembler [39], and then the $\mathrm{cv}$ Tombul $\mathrm{cp}$ genome constructed by NOVOplasty and the KX822768 cp genome were mapped to these contigs of $\mathrm{cv}$ Tombul genome using BLAST. Multiple contigs from the whole genome assembly matched the chloroplast sequences, but they were overlapping and fragmented (data not shown). Therefore it was concluded that using an assembler specialized for organellar genomes is advantageous for cp genome construction; further analysis was carried out using the first 161,667 bp of the genome assembly obtained from NOVOplasty, which also showed high similarity to the KX822768 cp genome.

The Tombul complete cp genome had a length of 161, $667 \mathrm{bp}$ and includes a pair of inverted repeats $26,368 \mathrm{bp}$ long, separated by a small and a large single copy region

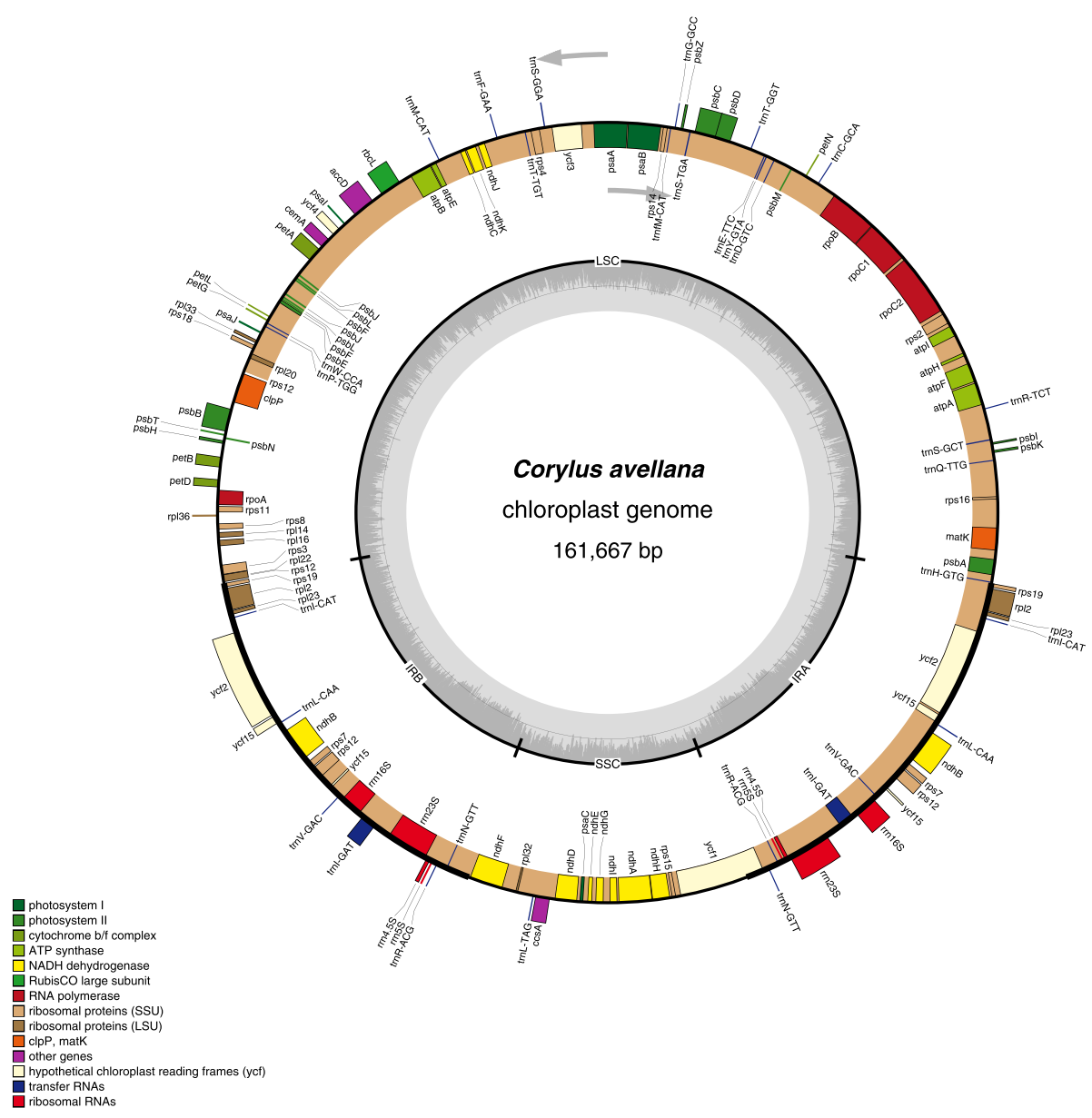

Fig. 1 The chloroplast genome map of Corylus avellana cv Tombul species. Genes lying outside the circle are transcribed in the counter clockwise direction, while those inside are transcribed in clockwise direction. The colored bars indicted different functional groups. The darker gray area in the inner circle denotes GC content while the lighter gray corresponds to the AT content of the genome. LSC, large single copy; SSC, small single copy; IR, inverted repeat 
of $18,733 \mathrm{bp}$ and $90,198 \mathrm{bp}$, respectively (Fig. 1). The overall GC content of $\mathrm{cv}$ Tombul cp genome was $36.40 \%$, and GC contents of the LSC and the SSC regions were 34.17 and $30.25 \%$, respectively. The GC content of the IR region was much higher than that of the LSC and SSC regions with $42.37 \%$, due to its relatively abundant GC-rich tRNA and rRNA genes.

For annotation of functional genes, three different prediction tools, namely GeSeq, cpGAVAS, and DOGMA, were compared (Fig. 2). These agreed with each other for the majority of the content and order of genes [40-43]. Generally, genes were included in the final map when at least 2 of the tools gave matching predictions. A total of 125 predicted functional genes were encoded within the Corylus avellana cv Tombul cp genome. Among them, 88 genes were unique, while 17 genes were duplicated in the IR region (IRA and IRB). Furthermore, the 105 distinct genes comprised 76 protein-coding, 25 tRNA and 4 rRNA genes. Seven protein coding genes ( $n d h B, r p l 2, r p l 23, r p s 7$, rps12, rps19, and $y c f 2$ ), six of the tRNA genes (trnI-CAT, trnI-GAT, trnL-CAA, trnN-GTT, trnR-ACG, and trnVGAC) and all rRNA genes (rrn16, rrn23, rrn5 and rrn4.5) were duplicated within the IR. Although the 3 annotation tools gave similar gene predictions a few differences were detected, especially in tRNA genes. The genes for trnATGC (duplicated in IR), trnK-TTT, trnL-TAA and $\operatorname{trn} V$ $T A C$ were only annotated by DOGMA, therefore they were not included in the final map. Fifty seven proteincoding genes and 18 tRNA genes were contained in the LSC region, while 12 protein-coding genes and one tRNA gene were identified in the SSC region. Three open reading frames (orf42, orf56, and orf188) and an addition hypothetical chloroplast reading frame $(y c f 68)$ were also identified with the DOGMA tool. Moreover, one gene,

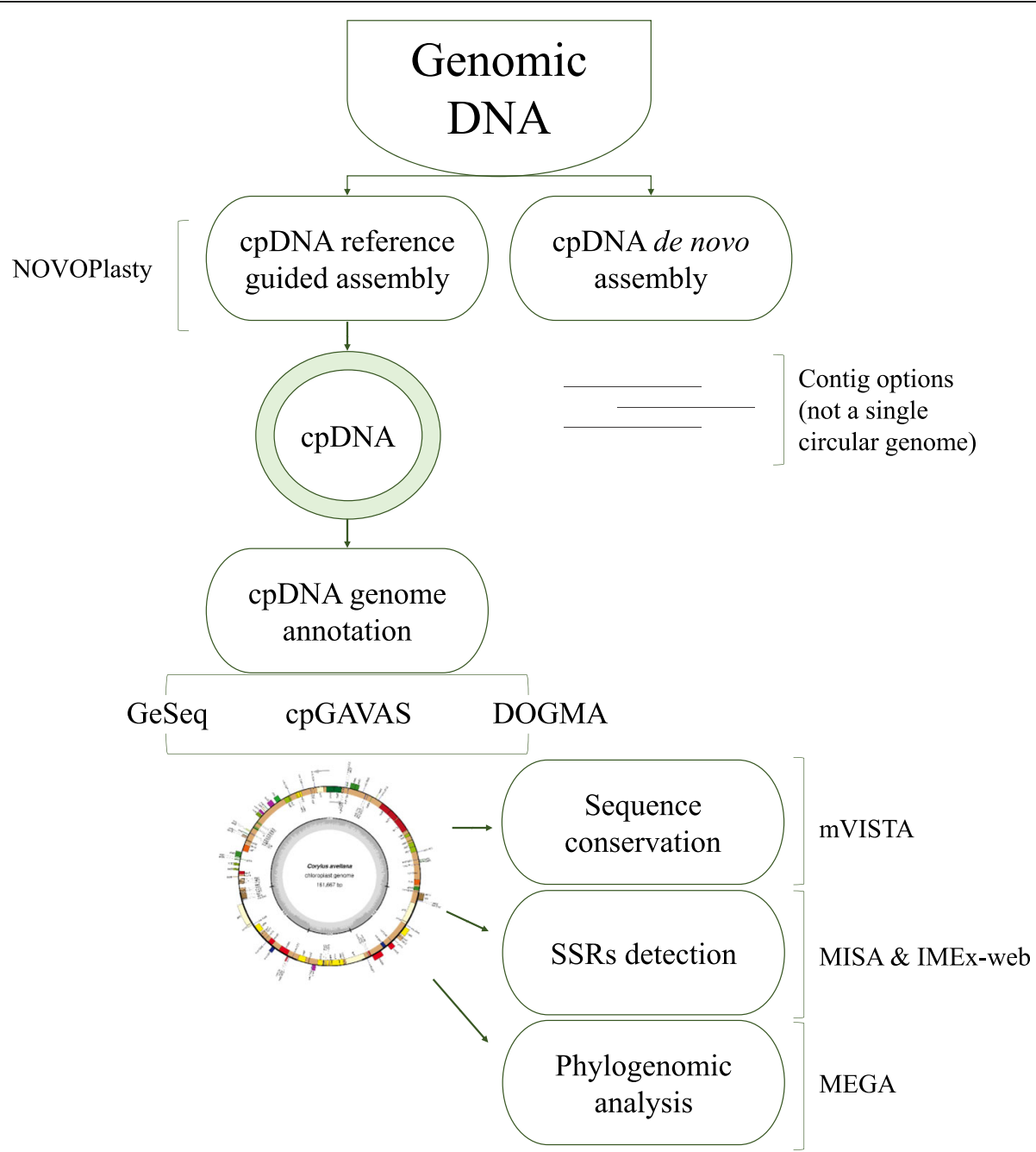

Fig. 2 Sequence alignment of 8 chloroplast genomes using mVISTA tool with Corylus avellana cv Tombul as a reference. Grey arrows above the alignment indicate the transcriptional directions of genes. Genome regions, exon and conserved non-coding sequences (CNS), are color coded as blue and red, respectively. Multiple alignment was carried by LAGAN option, and a cut-off of 50\% identity was used for the plots. The Y-axis indicated the percent identity between 50 and $100 \%$ 
$y c f 1$ located in the IRA/SSC junction, extended the IRA region by several bases. A $y c f$-like gene was also reported in the IRB region, one of the two IRs, with two annotation tools, DOGMA and GeSeq, but it was a truncated fragment of $y c f 1$ gene, and thus not included in the genome map. Of the 76 unique protein-coding genes, five genes (atpF, ndhA, ndhB, rpl2, and rpoC1) contained one intron, while two protein-coding genes ( $c l p P$ and $y c f 3)$ contained two introns each. The gene rps12 was annotated as transspliced gene of which the $5^{\prime}$-end exon was located in the LSC region while its intron and 3'- end exon were situated in the IR region (Additional file 1: Tables S1, S2).

RNA editing, a post-transcriptional modification process, exists in chloroplasts to encode appropriate amino acids and maintain conserved protein functions by correcting codons, especially by alteration of nucleotides from cytosine to uracil (C-to- $\mathrm{U})$ and less frequently from uracil to cytosine (U-to-C) [44-46]. Wang et al. [47] indicated that several changes were observed in protein-coding transcripts from chloroplasts, including $\mathrm{C}$ to $\mathrm{U}$, along with $\mathrm{G}$ to $A$ and $C$ to $G, A$ to $G$ and $G$ to A. Several nucleotide alterations are required to provide functional start codons in a handful of the genes annotated in the present study (Additional file 1: Table S3). RNA editing at these sites has not previously been confirmed in the Betulaceae, thereby further RNA sequence analysis should be carried out to determine whether these modifications occur.

Comparing the results of the annotation tools, ten genes (atpF, clpP, ndhA, ndhB, ndhK, petA, rpl2, rpoC1, $y c f 3, y c f 15)$ were erroneously reported twice as 2 gene fragments by DOGMA and GeSeq, whereas they were correctly reported as a single gene containing an intron by cpGAVAS (Additional file 1: Table S9). When the annotated genes were compared with those previously reported in other species' chloroplast sequences, the GeSeq tool gave the most accurate results for gene locations, including starting and end points of the CDS. DOGMA did not define the start and end point of exons, therefore start and stop codons had to be manually checked, and added from the cp genome. All of the genome and annotation information is shown in Fig. 1.

Prediction of cv Tombul cp gene functions was based on homology, and as expected they were mostly involved in photosynthesis and other metabolic processes. The genes were classified into three broad categories based on their functions: photosynthesis, self-replication and other genes. While 42 protein-coding genes participated in photosynthesis, 25 protein-coding genes were

Table 1 Gene contents and functional classification of cv Tombul chloroplast genome

\begin{tabular}{|c|c|c|c|}
\hline Category & Group of genes & Code of genes & List of genes \\
\hline \multirow[t]{6}{*}{ Genes for photosynthesis } & Subunits of ATP synthase & atp & atpA, atpB, atpE, atpF, atpH, atpl \\
\hline & Subunits of NADH-dehydrogenase & ndh & $\begin{array}{l}\text { ndhA, ndhB, ndhC, ndhD, ndhE, ndhF, } \\
\text { ndhG, ndhH, ndhl, ndhJ, ndhK }\end{array}$ \\
\hline & Subunits of cytochrome b/f complex & pet & petD, petG, petL, petN \\
\hline & Subunits of photosystem I & psa & psaA, psaB, psaC, psal, psaJ \\
\hline & Subunits of photosystem ॥ & psb & $\begin{array}{l}\text { psbA, psbB, psbC, psbD, psbE, psbF, psbH, } \\
\text { psbl, psbJ, psbK, psbL, psbM, psbN, psbT, psbz }\end{array}$ \\
\hline & Subunit of rubisco & rbc & $\mathrm{rbcL}$ \\
\hline \multirow[t]{5}{*}{ Self-replication } & Large subunit of ribosome & rpl & $\begin{array}{l}\text { rpl2,rpl14,rpl16, rpl20, rpl22, rpl23, rpl32, } \\
\text { rpl33, rpl36 }\end{array}$ \\
\hline & DNA dependent RNA polymerase & rpo & rpoA, rрoB, rрoC1, rpoC2 \\
\hline & Small subunit of ribosome & rps & $\begin{array}{l}\text { rps2, rps3, rps4, rps7, rps8, rps11, rps12, rps14, } \\
\text { rps15, rps16, rps18, rps19 }\end{array}$ \\
\hline & rRNA Genes & rrn & rrn4.5S, rrn5S, rrn16S, rrn23S \\
\hline & tRNA Genes & $\operatorname{trn}$ & $\begin{array}{l}\operatorname{trnC}-G C A \text {, trnD-GTC, trnE-TTC, trnF-GAA, trnfM-CAT, } \\
\text { trnG-GCC, trnH-GTG, trnM-CAT, trnP-TGG, trnQ-TTG, } \\
\text { trnR-TCT, trnS-GCT, trnS-GGA, trnS-TGA, trnT-GGT, } \\
\text { trnT-TGT, trnW-CCA, trnY-GTA, trnL-TAG, trnl-CAT, } \\
\text { trnl-GAT, trnL-CAA, trnN-GTT, trnR-ACG, trnV-GAC }\end{array}$ \\
\hline \multirow[t]{5}{*}{ Other genes } & Subunit of Acetyl-CoA-carboxylase & acc & $\mathrm{accD}$ \\
\hline & c-type cytochrome synthesis gene & cCs & $\operatorname{ccs} A$ \\
\hline & Envelop membrane protein & cem & cemA \\
\hline & Protease & $\mathrm{clp}$ & $\mathrm{clpP}$ \\
\hline & Maturase & mat & matk \\
\hline Genes of unkown function & Conserved open reading frames & ycf & $y c f 1, y c f 2, y c f 3, y c f 4$ \\
\hline
\end{tabular}


involved in the chloroplast self-replication processes, and 5 genes represented other functions, all of which were summarized in Table 1.

Based on a sequence similarity search of the whole genome, the $C$. avellana $\mathrm{cv}$ Tombul chloroplast was most similar to chloroplast genomes belonging to the Corylus family with a range from 99.46 (Corylus wangii, Accesion: MH628454.1) to 99.88\% (Corylus heterophylla var. sutchuenensis, Accesion: MF996573.1) identity via Basic Local Alignment Search Tool (BLAST) search in NCBI website (http://blast.ncbi.nlm.nih.gov/) against Viridiplantae (taxid: 33090) [38]. In addition, Carpinus and Ostrya families also showed high similarity with cv Tombul cp genome with nearly 98.91 and $99.21 \%$ identity, respectively. (Additional file 1: Table S4).

\section{Comparison of chloroplast genome sequences with other species}

The similarities and differences of the cp genome between $C$. avellana $\mathrm{cv}$ Tombul and other species, including representatives of the Malpighiales, Fabales and Brassicales, were determined by a global alignment program, mVISTA [48]. The chloroplast genome sequences were aligned to each other and plotted using $C$. avellana $\mathrm{cv}$ Tombul as a reference (Fig. 3). Tombul had a similar $\mathrm{cp}$ genome size to the other species, which range from 152,217 bp to $161,303 \mathrm{bp}$ (Tombul cp genome size is $161,667 \mathrm{bp}$ ). In addition, the alignment revealed a very high level of identity in the global patterns of sequence similarities with KX822768, an accession of an unspecified C. avellana variety found in China, and Betula nana with 99.8 and $96.6 \%$ identity, respectively. As expected, coding regions were more highly conserved than non-coding regions. The highest polymorphism was observed in intergenic regions (such as rps16-psbK, $p s b I$-atpA, $p s b M-p s b D$ ), but the $y c f 1$ gene had higher variability regions, especially between distant species. At the species level, nucleotide substitution could more rapidly occur in intergenic regions, and these regions with high levels of divergence could have high potential for

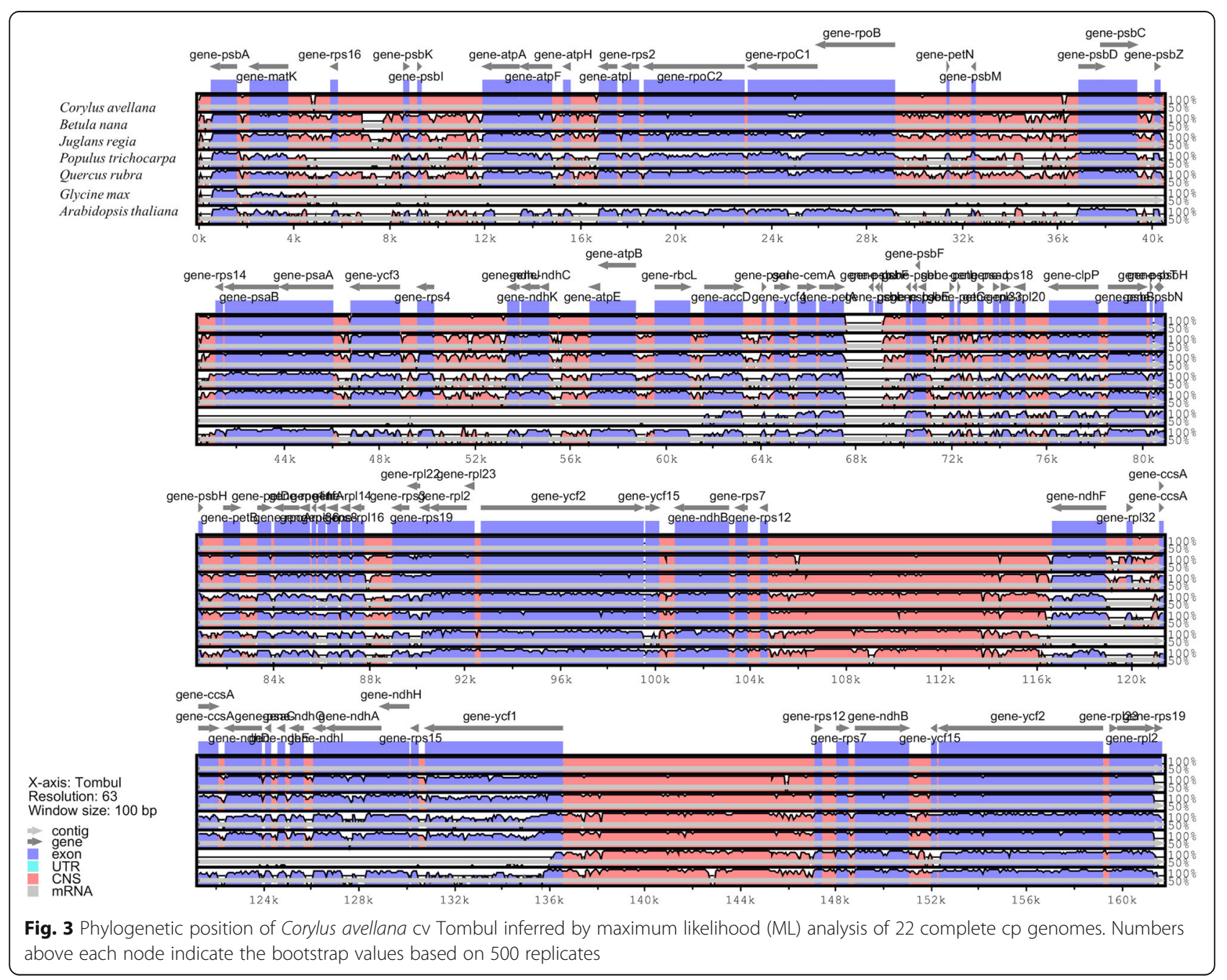


developing molecular markers for population genetic analysis between varieties. Furthermore, a region was detected in the $\mathrm{cv}$ Tombul cp genome from $\sim 68$ to $69 \mathrm{~kb}$ that was conserved with KX822768 but none of the other species presented in the global alignment. This region contained duplicates of the $p s b F, p s b J$ and $p s b L$ genes from the adjacent region, and an unprocessed petA gene. This could be a tandem duplication specific to the hazelnut lineage; further Corylus chloroplast genomes should be explored to determine whether it is found in other species from this genus.

\section{SSR analysis}

Simple sequence repeats (SSR) are useful in characterization of genetic diversity. According to the MISA web tool, a total of 83 SSRs were identified in the $\mathrm{cv}$ Tombul cp genome [49]. Among these SSRs, there were 44, 19, 4, 13, 2, 1 for mono-, di-, tri-, tetra- and penta- nucleotide repeats, respectively (Additional file 1: Table S5). The largest proportion of simple repeats was classified as mononucleotides (48.2\%). While most of the mononucleotides were composed of A/T (90.9\%), most of the dinucleotides were AT/ TA (84.2\%) (Additional file 1: Figure S1). Similar results were obtained from IMEx-web server [50]. Only a few differences were shown in the direction of SSRs (Additional file 1: Table S6). These SSR regions may be useful in developing markers useful to elucidate genome evolution and chloroplast rearrangements among species.

\section{Phylogeny inference}

The complete $\mathrm{cp}$ genome sequences of 22 species from Fagales order were obtained from the NCBI and used for phylogenetic analysis, including representatives of genera of Betulaceae, Fagaceae, and Juglandaceae. As chloroplast protein sequences showed high similarity among related species, the phylogenetic analysis was carried out using the whole cp genome sequences. Tree construction was carried by the maximum likelihood method with 500 replicates. All nodes of these phylogenetic trees were strongly supported by bootstrap values (BS). The 22 taxa were classified into four major clades. A monophyletic group was observed incorporating the Corylus, Betula and Juglans species. Fagus and the sister group of Quercus and Castanopsis were located at the basal position. Moreover, within the Betulaceae, Carpinus and Ostrya clustered into a clade which was the sister to the clade Corylus and showed greater divergence from the clade formed by Betula species. As stated in the literature, Corylus was closest to Carpinus and Ostrya species, and then relatively close to Betula, which is consistent with their taxonomic classification but provides greater insight into the relatedness of these genera (Fig. 4) [51, 52].

In the clade Corylus, 6 species were divided into four subclades. C. wangii was located at the basal position, while C. mandshurica and C. heterophylla clustered into a sister group, while $C$. fargesii and $C$. chinensis clustered together. The phylogenetic tree indicated that cv Tombul, although it formed a distinct subclade, exhibited a closer relationship with $C$. fargesii and $C$. chinensis than the other varieties (Fig. 4) [53, 54].

\section{Discussion \\ Comparison of methods for assembling cp sequences from WGS data}

The assembly of cp genomes from whole genome shotgun (WGS) sequences is a useful strategy for characterizing cp genes, structure, function and phylogenetic relationships. Multiple tools have been developed to construct and annotate cp genomes. This study reported a complete cp genome sequence of Corylus avellana cv Tombul, annotated by different available annotation tools. Initially, the de novo assembler NOVOPlasty was used to reconstitute the Tombul cp genome (Fig. 2) [37]. A single 200,017 bp contig was obtained from raw WGS sequences by NOVOplasty. The comparison of the contig with the KX822768 cp genome, published in GenBank, indicated that the last part of the sequence, (161,667-200,017 bp), was nearly identical to other segments of the Tombul chloroplast genome. Nanopore sequencing reads belonging to $\mathrm{cv}$ Tombul, were aligned to the contig, and a subset of reads matched these additional parts. Therefore, we considered the possibility that the $\mathrm{cp}$ genome of $\mathrm{cv}$ Tombul could be physically larger than the reported $C$. avellana cp genome. However, BLAST results indicated that this part consisted of two segments, each of which was $100 \%$ identical to a region in the first $161 \mathrm{~kb}$ of the $\mathrm{cv}$ Tombul $\mathrm{cp}$ genome (Additional file 1: Figure S2) [47]. Furthermore, the mapped read depth of the duplicated segments was approximately half of that of the rest of the cp genome. Hence, we concluded that the additional $39 \mathrm{~kb}$ was an artefact of the NOVOplasty assembly algorithm. Further analysis was carried out using the first $161,667 \mathrm{bp}$ of the genome assembly.

\section{Comparison of methods for annotation of $\mathrm{cp}$ genome for cv Tombul}

The cv Tombul cp genome presented similar characteristics to other angiosperm cp genomes. In addition, it exhibited some differences between closely related species. There is a previously reported sequence for $C$. avellana deposited in Genbank (KX822768), cultivated in China, but no varietal information was provided for this accession. While the general characteristics of cv Tombul cp genome are highly consistent with KX822768, a few differences were detected at the gene level. Two genes, atpF and $\operatorname{clpP}$, were reported as unprocessed in the older sequence, however full-length protein 


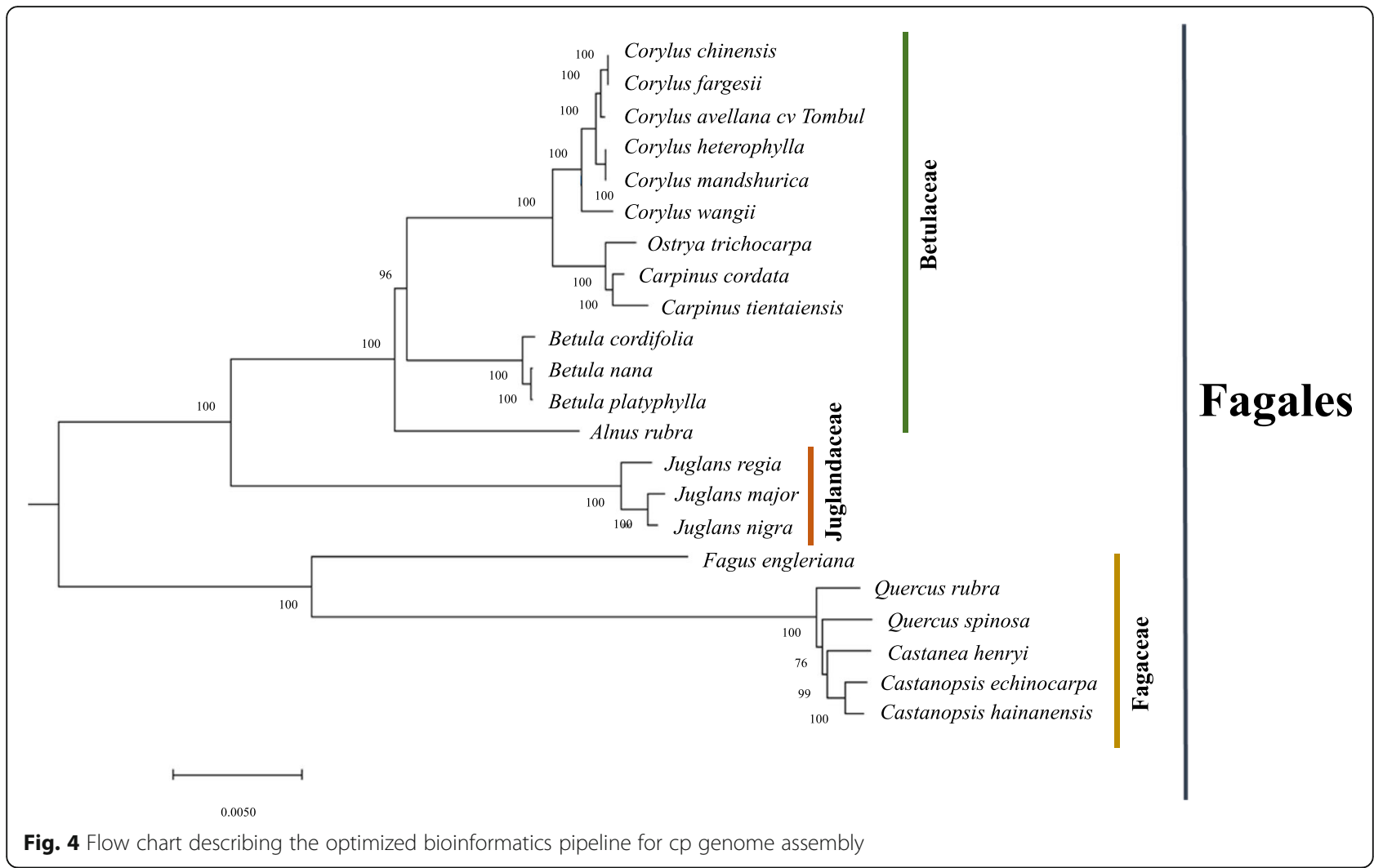

sequences were predicted for these genes in the cv Tombul cp genome. Furthermore, the genes $a c c D$, $p s b M$, and trnI-GAT, were not annotated in KX822768, but they were present in the $\mathrm{cv}$ Tombul cp genome. Lastly, $p s b F$, $p s b J$ and $p s b L$ genes were found twice in the cr Tombul cp genome (Additional file 1: Table S7). The length of the $\mathrm{cv}$ Tombul $\mathrm{cp}$ genome was found to be similar to other Corylus and Quercus species, but a difference was indicated with Populus and Juglans species [53-57]. Although the length differed among species, the GC contents were very similar in angiosperm cp genomes (Additional file 1: Table S8) [58].

Two hypothetical chloroplast protein coding sequences, $y c f 15$ and $y c f 68$, were also identified in our annotation process. Whereas they were represented as functional protein-coding genes in some studies [59], they were classified as pseudogenes in our map because of containing several internal stop codons in their coding sequences, consistent with observations in several other species $[7,60]$. Moreover, the gene $y c f 15$ was annotated between rps7 and trnV-GAC in some studies, while some others reported that it was located between $y c f 2$ and $\operatorname{trnL}-C A A[61,62]$. In the present study, the gene $y c f 15$ was detected in both positions, thereby further studies are required to clarify whether either of these is expressed.
The annotation of the cv Tombul cp genome was carried out using three different tools, cpGAVAS, DOGMA and GeSeq [40-43]. In terms of gene content, similar results were obtained from all tools. A number of genes were annotated as fragmented by DOGMA and GeSeq, whereas they were found as a single gene in cpGAVAS containing introns in specific locations. Additionally, more tRNA genes were detected using the DOGMA tool than the other two (Additional file 1: Table S9). The GeSeq annotation tool provides more precise results for gene locations, which may be because it chooses a broad range of BLAT reference sequences, including closely related taxa. Because it is not suitable for defining the start and end of exons, the DOGMA annotation needs manual editing, and additionally the identification of the IR region was not supported by this tool. CpGAVAS results showed high similarity with the GeSeq findings. If a cp genome belonging to a closely related taxon is available, the GeSeq annotation tool is the most useful for the analysis. In other cases, annotation with both GeSeq and cpGAVAS, followed by comparison of the results from both tools, provides the most precise information about functional genes and locations with minimal configuration.

Highly conserved structure, similar gene content and order were determined by comparative genome analysis of $\mathrm{cv}$ Tombul genome with seven different 
species, indicating that $\mathrm{cv}$ Tombul $\mathrm{cp}$ genome contains largely the same coding genes, tRNAs and rRNAs. However, the length of the $\mathrm{cp}$ genome from cv Tombul slightly differed from the published sequence KX822768 in GenBank, from which it could be inferred that some genetic differences exist even between cultivars.

\section{Repeat analysis}

Simple sequence repeats (SSR) are useful for characterization of genetic diversity and development of molecular markers for phylogenetic studies and breeding. Herein, we identified several microsatellite sequences in the $C$. avellana $\mathrm{cv}$ Tombul cp genome, most of which were distributed in the intergenic regions, although some SSRs were detected in coding genes. Multiple SSR types were detected in the $y c f 1$ protein-coding gene, confirming the results of comparative genome analysis that $y c f 1$ contains high variability regions. The majority of simple repeats from the $\mathrm{cv}$ Tombul cp genome were classified as mononucleotides and dinucleotides, which were mainly composed of adenine (A) or thymine (T) repeats, and rarely contained guanine $(G)$ or cytosine $(C)$. Our findings presented similar results to previous studies $[7,63,64]$. These features could provide deeper information for phylogenetic research of Corylus by allowing development of species- and variety-specific molecular markers.

\section{Phylogenetic analysis}

Previous studies have resolved the relationships of Betulaceae family, and most taxonomists have agreed that the Betulaceae family is divided into two subfamilies, named as Betuloideae (genera: Alnus and Betula) and Coryloideae (genera: Corylus, Carpinus, Ostrya, and Ostryopsis) [51]. The generic relationships within Coryloideae were studied by molecular markers including $m a t K$ and $r b c L$ genes, and ITS regions [51, 65-67]. We found a similar grouping within the cp genome phylogeny, including that Corylus formed a monophyletic group and had a close relationship with Carpinus and Ostrya: the results of our analyses supported the previous studies [52]. Ostrya formed a sister group with Carpinus, and these genera together constituted a sister group to Corylus. Whereas relationships between subfamilies have been fairly well resolved, the inter-specific relationships within Corylus have not been completely determined due to lack of information about taxon sampling for Corylus species, and limited studies at the molecular level [29]. Our results about the relationship of Corylus species supported those recently reported by Yang et al. [7]; Corylus avellana cv Tombul exhibited a close relationship to the sister group of $C$. fargesii and C. chinensis, which are tree species in Corylus family, and the most distant relationship to the primitive species, C. wangii. As briefly stated, the complete cp genomes provide more in-depth information about both inter- and intraspecific relationships, and for evolutionary studies.

\section{Conclusion}

Corylus is a phylogenetically and economically important genus with 16-20 species, in the family Betulaceae. Because of commercial and ornamental values of Corylus species, greater study of this genus can contribute to both science and the economy. In this study, we assembled the $C$. avellana cv Tombul cp genome by using WGS sequences generated as part of a whole genome sequencing project. The $\mathrm{cp}$ genome of $\mathrm{cv}$ Tombul has a typical cp genome structure, and is highly similar to other cp genomes of the Betulaceae family. Our results confirm that complete and highly-accurate chloroplast genome assemblies can be simply obtained from next generation whole genome shotgun data, but that assembly and annotation tools must be carefully selected and cross-checked for potential errors. Although the results were similar in all annotation tools in terms of gene content, GeSeq and cpGAVAS provided better results for gene locations. If the location information of exons and introns of a gene was needed for further analysis, annotation should be carried by using GeSeq. According to phylogenetic analysis, it was observed that Corylus avellana cv Tombul had a close relationship to C. fargesii and $C$. chinensis. Therefore, these two Corylus species may be especially useful for crossbreeding and grafting with $C$. avellana, in order to produce resilient and productive varieties. Moreover, the interspecific relationships of genus Corylus could be more precisely understood with enhanced taxon sampling. In the future, we are considering wider $\mathrm{cp}$ genome sampling of other cultivated varieties, to investigate whether cultivar specific markers exist or not, and the development of molecular markers for deeper information about phylogeny.

\section{Methods \\ DNA extraction and sequencing}

DNA extraction and sequencing was carried out as part of an ongoing $C$. avellana genome sequencing project. High molecular weight DNA was extracted from young leaf buds using a CTAB method optimized for Betulaceae [68]. Whole genome shotgun libraries were prepared using TruSeq kits and selected for an insert size of 600-800 nt. Paired-end sequencing was carried out on a Illumina HiSeq4000 and reads were deposited in the European Nucleotide Archive (Project accession: PRJEB31933). NanoPore sequencing reads were also obtained for the same cv Tombul genome project. NanoPore sequencing was carried out on the MinION platform using R9.4 flowcells and Ligation Sequencing 
Kit 1D, according to the manufacturer's protocols (Oxford NanoPore Technologies, Oxford, UK).

\section{Chloroplast genome assembly and annotation}

Whole genome Illumina paired-end raw data without adapters were used in de novo assembler NOVOPlasty, a seed-extend based assembler [37] (Fig. 2). The cp genome was assembled from WGS data, initiated by a seed sequence, which is iteratively extended bidirectionally, to obtain the circular genome. Using a reference genome is optional in the pipeline, but can be useful to obtain a single circular genome, and to eliminate manual adjustments. In this study, Arabidopsis thaliana (KX551970.1) and Corylus avellana complete cpDNA sequences (KX822768.2) were used as seed and reference genomes, respectively. We specified the following parameters: automatic insert size detection, a genome size range from 120,000 to 200,000 , a K-mer value of 39 , an insert range of 1.8, a strict insert range of 1.3, and the pairedend reads option. Moreover, the contig was checked using BLAST searches against the available complete $\mathrm{cp}$ sequence of KX822768 [47]. Relative positions were manually curated according to the reference genome, and the complete cp genome for Tombul cultivar was finally acquired for further analysis. In addition, Illumina paired-end raw sequence reads were processed by Trimmomatic to remove adapters, and trimmed sequences were assembled using ABySS $1.9[39,69]$. Then, the cv Tombul cp genome obtained from NOVOplasty was aligned to the ABySS contigs using BLAST.

The Tombul cp genome was annotated through three different online programs, GeSeq, CpGAVAS and DOGMA with default parameters [40-43]. For the annotation file, the gene locations were compared and accepted when they matched the same position with at least two annotation tools. MEGA pairwise alignment was additionally used to confirm the genes among closely related taxa, and the gene locations were verified from cv Tombul cp genome sequences. Protein-coding and tRNA genes found by only one tool were not included in the map. The visual image of annotation was illustrated with the help of OGDRAW [70]. The final assembly was submitted to GenBank (MN082371).

\section{Comparative chloroplast genomic analysis}

Complete cp genomes of seven species, including Corylus avellana (GenBank accession number: KX822768.2), Betula nana (GenBank accession number: NC_033978.1), Juglans regia (GenBank accession number: MF167463.1), Populus trichocarpa (GenBank accession number: EF489041.1), Quercus rubra (GenBank accession number: JX970937.1), Glycine max (GenBank accession number: NC_007942.1) and Arabidopsis thaliana (GenBank accession number: KX551970.1), were downloaded from NCBI, in order to compare the overall similarities among different $\mathrm{cp}$ genomes with Tombul cultivar. Pairwise alignments were implemented in the LAGAN alignment program included in mVISTA program with default parameters [48] using the annotation of Corylus avellana cv Tombul (Betulaceae, Fagales; GenBank accession number: MN082371) as the reference.

\section{SSR analysis}

Simple sequence repeats (SSRs) were detected using two different microsatellite identification web tools, MISA (MIcroSAtellite identification tool) and IMEx-web (Imperfect Microsatellite Extraction Webserver) by setting the minimum number of repeats to $10,5,4,3,3$ and 3 for mono-, di-, tri-, tetra-, penta- and hexanucleotides, respectively $[49,50]$.

\section{Phylogenomic analysis}

The complete cp genome sequences of 22 species from Fagales were used for phylogenetic analysis, including representatives of genera from the Betulaceae, Fagaceae, and Juglandaceae. The $\mathrm{cp}$ genomes of species were aligned with multiple sequence alignment tool, MUSCLE [71]. All sequence gaps were excluded after alignment in the analysis. The evolutionary history was inferred by using the Maximum Likelihood method and TamuraNei model and analyses were conducted in MEGA X $[72,73]$. The bootstrap consensus tree inferred from 500 replicates was taken to represent the evolutionary history of the taxa analyzed. All positions with less than $90 \%$ site coverage were eliminated, i.e., fewer than $10 \%$ alignment gaps, missing data, and ambiguous bases were allowed at any position (partial deletion option).

\section{Supplementary information}

Supplementary information accompanies this paper at https://doi.org/10. 1186/s12864-019-6253-5.

Additional file 1: Table S1. Comparison of three different annotation tools in terms of protein-coding gene content. Table S2. Comparison of three different annotation tools in terms of transfer and ribosomal RNA gene content. Table S3. Nucleotide changes in protein-coding genes. Table S4. BLAST result of the cV Tombul chloroplast genome against Viridiplantae (best 100 hits). Table S5. Simple sequence repeats within the cv Tombul chloroplast genome. Table S6. Comparison of two SSR identification tools for the cV Tombul chloroplast genome. Table S7. Differences between cV Tombul and KX822768 cp genome published in GenBank. Table S8. The features of Fagales and Malpighiales plastomes. Table S9. Differences between annotation tools. Figure S1. Number of classified SSR repeat types (considering complementary sequences). Figure S2. Schematic that explains the structure of cV Tombul chloroplast genome.

\section{Abbreviations}

A: Adenine; AFLP: Amplified fragment length polymorphism; BS: Bootstrap; C: Cytosine; cp: Chloroplast; cpDNA: Chloroplast DNA; Cv: Cultivar; G: Guanine; IR: Inverted repeat; ISSR: Inter simple sequence repeats; ITS: Internal transcribed spacer; LSC : Large single copy; RAPD: Random amplified polymorphic DNA; SSC: Small single copy; SSR: Simple sequence repeat; T: Thymine; WGS: Whole genome shotgun 


\section{Acknowledgments}

This study utilized the Sabanci HPC Cluster for computing support. The authors would like to thank Bihter Avsar and lipek Bilge for helpful discussions.

\section{Authors' contributions}

KK developed methods, analyzed the data and drafted the manuscript. SJL conceived the study, generated sequencing data and revised the manuscript. Both authors read and approved the final manuscript.

\section{Funding}

This study was supported by the Scientific and Technological Research Council of Turkey (TÜBITAK), grant no. 2150446.

\section{Availability of data and materials}

The raw sequencing data used in this study are available in the ENA repository (project accession: PRJEB31933; https://www.ebi.ac.uk/ena/data/ view/PRJEB31933). The full C. avellana cv. Tombul chloroplast assembly and annotation is available in the Genbank repository (Accession no: MN082371; https://www.ncbi.nlm.nih.gov/nuccore/MN082371).

\section{Ethics approval and consent to participate}

Not applicable

\section{Consent for publication}

Not applicable

\section{Competing interests}

The authors declare that they have no competing interests.

\section{Author details}

${ }^{1}$ Faculty of Engineering and Natural Sciences, Sabanci University, 34956 Istanbul, Turkey. ${ }^{2}$ Sabanci University Nanotechnology Research and Application Centre (SUNUM), Sabanci University, 34956 Istanbul, Turkey.

Received: 15 July 2019 Accepted: 31 October 2019

Published online: 20 November 2019

\section{References}

1. Casus-Agustench P, Salas-Huetos A, Salas-Salvado J. Mediterranean nuts: origins, ancient medicinal benefits, and symbolism. Public Health Nutr. 2011; 14:2296-301.

2. USDA. Basic Report: 12120, Nuts, hazelnuts or filberts: USDA Agricultural Research Service; 2014. http://ndb.nal.usda.gov/ndb/foods/show/3710. Last access date: 7 July 2015

3. Rowley ER, VanBuren R, Bryant DW, Priest HD, Mehlenbacher SA, Mockler TC. A draft genome and high-density genetic map of European hazelnut (Corylus avellana L.). BioRxiv. 2018. https://doi.org/10.1101/469015.

4. Lee SB, Kaittanis C, Jansen RK, Hostetler JB, Tallon LJ, Town CD, Daniell H. The complete chloroplast genome sequence of Gossypium hirsutum: organization and phylogenetic relationships to other angiosperms. BMC Genomics. 2006:23:7-61.

5. Green BR. Chloroplast genomes of photosynthetic eukaryotes. Plant J. 2011; 66:34-44.

6. Daniell H, Lin CS, Yu M, Chang WJ. Chloroplast genomes: diversity, evolution, and applications in genetic engineering. Genome Biol. 2016. https://doi.org/10.1186/s13059-016-1004-2.

7. Yang Z, Zhao T, Ma Q, Liang L, Wang G. Comparative genomics and phylogenetic analysis revealed the chloroplast genome variation and interspecific relationships of Corylus (Betulaceae) species. Front Plant Sci. 2018. https://doi.org/10.3389/fpls.2018.00927.

8. Suo Z, Zhang C, Zheng Y, He L, Jin X, Hou B, et al. Revealing genetic diversity of tree peonies at micro-evolution level with hypervariable chloroplast markers and floral traits. Plant Cell Rep. 2012. https://doi.org/10. 1007/s00299-012-1330-0

9. Dong W, Xu C, Li D, Jin X, Li R, Lu Q, et al. Comparative analysis of the complete chloroplast genome sequences in psammophytic Haloxylon species (Amaranthaceae). PeerJ. 2016. https://doi.org/10.7717/peerj.2699.

10. Wang M, Xie X, Yan B, Yan X, Luo J, Liu Y, et al. The completed chloroplast genome of Ostrya trichocarpa. Conserv Genet Resour. 2017. https://doi.org/ 10.1007/s12686-017-0869-z.
11. Xu C, Dong WP, Li WQ, Lu YZ, Xie XM, Jin XB, et al. Comparative analysis of six Lagerstroemia complete chloroplast genomes. Front Plant Sci. 2017. https://doi.org/10.3389/fpls.2017.00015.

12. Percy DM, Argus GW, Cronk QC, Fazekas AJ, Kesanakurti PR, Burgess KS, et al. Understanding the spectacular failure of DNA barcoding in willows (Salix): does this result from a trans-specific selective sweep. Mol Ecol. 2014; 23:4737-56

13. Jansen RK, Cai Z, Raubeson LA, Daniell H, de Pamphilis CW, Leebens-Mack J, et al. Analysis of 81 genes from 64 plastid genomes resolves relationships in angiosperms and identifies genome-scale evolutionary patterns. Proc Natl Acad Sci U S A. 2007:104:19369-74.

14. Moore MJ, Bell CD, Soltis PS, Soltis DE. Using plastid genome scale data to resolve enigmatic relationships among basal angiosperms. Proc Natl Acad Sci U S A. 2007;104:19363-8.

15. Moore MJ, Soltis PS, Bell CD, Burleigh JG, Soltis DE. Phylogenetic analysis of 83 plastid genes further resolves the early diversification of eudicots. Proc Natl Acad Sci U S A. 2010;107:4623-8

16. Drouin $\mathrm{G}$, Daoud H, Xia J. Relative rates of synonymous substitutions in the mitochondrial, chloroplast and nuclear genomes of seed plants. Mol Phylogenet Evol. 2008. https://doi.org/10.1016/j.ympev.2008.09.009.

17. Parks $\mathrm{M}$, Cronn $\mathrm{R}$, Liston $\mathrm{A}$. Increasing phylogenetic resolution at low taxonomic levels using massively parallel sequencing of chloroplast genomes. BMC Biol. 2009;7:84.

18. Carbonell-Caballero J, Alonso R, Ibañez V, Terol J, Talon M, Dopazo J. A phylogenetic analysis of 34 chloroplast genomes elucidates the relationships between wild and domestic species within the genus Citrus. Mol Biol Evol. 2015;32:2015-35.

19. Smith DR. Mutation rates in plastid genomes: they are lower than you might think. Genome Biol Evol. 2015. https://doi.org/10.1093/gbe/evv069.

20. Xi Z, Ruhfel BR, Schaefer H, Amorim AM, Sugumaran M, Wurdack KJ, et al. Phylogenomics and a posteriori data partitioning resolve the cretaceous angiosperm radiation Malpighiales. Proc Natl Acad Sci U S A. 2012;109: 17519-24.

21. Barrett CF, Davis Jl, Leebens-Mack J, Conran JG, Stevenson DW. Plastid genomes and deep relationships among the commelinid monocot angiosperms. Cladistics. 2013;29:65-87.

22. Galderisi U, Cipollarom A, Bernardo G, De Masi L, Galano G, Cascino A. Identification of hazelnut (Corylus avellana) cultivars by RAPD analysis. Plant Cell Rep. 1999. https://doi.org/10.1007/s002990050637.

23. Zhao S, Su SC, Chen ZG, Shuyan WZ. An assessment of the genetic diversity and population genetic structure concerning the Corylus heterophylla Fisch., grown in the Tieling district of Liaoning province, using SSR markers. J Fruit Sci. 2015. https://doi.org/10.13925/j.cnki.gsxb.20150187.

24. Beltramo C, Valentini N, Portis E, Torello Marinoni D, Boccacci P, Sandoval Prando MA, et al. Genetic mapping and QTL analysis in European hazelnut (Corylus avellana L.). Mol Breed. 2016. https://doi.org/ 10.1007/s11032-016-0450-6.

25. Di XY, Liu KW, Hou SQ, Ji PL, Wang YL. Genetic variation of hazel (Corylus heterophylla) populations at different altitudes in Xingtangsi forest park in Huoshan. Plant Omics J. 2014;7:213-20.

26. Essadki M, Ouazzani N, Lumaret $R$, Moumni M. ISSR variation in olivetree cultivars from Morocco and other western countries of the Mediterranean Basin. Genet Resour Crop Evol. 2006. https://doi.org/10. 1007/s10722-004-1931-8.

27. Ferreira JJ, Garcia C, Tous J. Structure and genetic diversity of local hazelnut collected in Asturias (Northern Spain) revealed by ISSR markers. ActaHortic. 2009. https://doi.org/10.17660/ActaHortic.2009.845.20.

28. Zong JW, Zhao TT, Ma QH, Liang LS, Wang GX. Assessment of genetic diversity and population genetic structure of Corylus mandshurica in China using SSR markers. PLoS One. 2015. https://doi.org/10.1371/ journal.pone.0137528.

29. Erdogan V, Mehlenbacher SA. Phylogenetic relationships of Corylus species (Betulaceae) based on nuclear ribosomal DNA ITS region and chloroplast matK gene sequences. Syst Bot. 2000. https://doi.org/10.2307/2666730.

30. Leinemann L, Steiner W, Hosius B, Kuchma O, Arenhövel W, Fussi B, et al. Genetic variation of chloroplast and nuclear markers in natural populations of hazelnut (Corylus avellana L.) in Germany. Plant Syst Evol. 2013. https:// doi.org/10.1007/s00606-012-0727-0.

31. Taberlet P, Coissac E, Pompanon F, Gielly L, Miquel C, Valentini A, et al. Power and limitations of the chloroplast trnL (UAA) intron for plant DNA barcoding. Nucleic Acids Res. 2007;35:e14. 
32. Kane N, Sveinsson S, Dempewolf H, Yang JY, Zhang D, Engels JMM, et al. Ultra-barcoding in cacao (Theobroma Spp.; Malvaceae) using whole chloroplast genomes and nuclear ribosomal DNA. Am J Bot. 2012;99:320-9.

33. Bakker FT, Lei D, Yu J, Mohammadin S, Wei Z, van de Kerke S, et al. Herbarium genomics: plastome sequence assembly from a range of herbarium specimens using an iterative organelle genome assembly pipeline. Biol J Linn Soc. 2016;117:33-43.

34. Wang X, Zhou T, Bai G, Zhao Y. Complete chloroplast genome sequence of Fagopyrum dibotrys: genome features, comparative analysis and phylogenetic relationships. Sci Rep. 2018. https://doi.org/10.1038/s41598-018-30398-6.

35. Osuna-Mascaró C, Rubio de Casas R, Perfectti F. Comparative assessment shows the reliability of chloroplast genome assembly using RNA-seq. Sci Rep. 2018. https://doi.org/10.1038/s41598-018-35654-3.

36. Vinga S, Carvalho AM, Francisco AP, Russo LM, Almeida JS. Pattern matching through chaos game representation: bridging numerical and discrete data structures for biological sequence analysis. Algorithms Mol Biol. 2012;7:10.

37. Dierckxsens N, Mardulyn P, Smits G. NOVOPlasty: de novo assembly of organelle genomes from whole genome data. Nucleic Acids Res. 2017. https://doi.org/10.1093/nar/gkw955.

38. Camacho C, Coulouris G, Avagyan V, Ma N, Papadopoulos J, Bealer K, Madden TL. BLAST+: architecture and applications. BMC Bioinformatics. 2009. https://doi.org/10.1186/1471-2105-10-421.

39. Simpson JT, Wong K, Jackman SD, Schein JE, Jones SJ, Birol I. ABySS: a parallel assembler for short read sequence data. Genome Res. 2009. https:// doi.org/10.1101/gr.089532.108.

40. Tillich M, Lehwark P, Pellizzer T, Ulbricht-Jones ES, Fischer A, Bock R, Greiner S. GeSeq - versatile and accurate annotation of organelle genomes. Nucleic Acids Res. 2017:45:W6-W11.

41. Liu C, Shi L, Zhu Y, Chen H, Zhang J, Lin X, Guan X. CpGAVAS, an integrated web server for the annotation, visualization, analysis, and GenBank submission of completely sequenced chloroplast genome sequences. BMC Genomics. 2012. https://doi.org/10.1186/1471-2164-13-715.

42. Shi $L$, Chen $H$, Jiang $M$, Wang $L$, Wu $X$, Huang L, Liu C. CPGAVAS2, an integrated plastome sequence annotator and analyzer. Nucleic Acids Res. 2019. https://doi.org/10.1093/nar/gkz345.

43. Wyman SK, Jansen RK, Boore JL. Automatic annotation of organellar genomes with DOGMA. Bioinformatics. 2004;22:3252-5.

44. Tillich M, Lehwark P, Morton BR, Maier UG. The evolution of chloroplast RNA editing. Mol Biol Evol. 2006. https://doi.org/10.1093/molbev/msl054.

45. Chateigner-Boutin AL, Small I. Plant RNA editing. RNA Biol. 2010. https://doi. org/10.4161/rna.7.2.11343.

46. Rodrigues NF, Christoff AP, da Fonseca GC, Kulcheski FR, Margis R. Unveiling Chloroplast RNA Editing events using next generation small RNA sequencing data. Front Plant Sci 2017; doi: https://doi.org/10.3389/ fpls.2017.01686.

47. Wang S, Yang C, Zhao X, Chen S, Qu GZ. Complete chloroplast genome sequence of Betula platyphylla: gene organization, RNA editing, and comparative and phylogenetic analyses. BMC Genomics. 2018. https://doi. org/10.1186/s12864-018-5346-X.

48. Frazer KA, Pachter L, Poliakov A, Rubin EM, Dubchak I. VISTA: computational tools for comparative genomics. Nucleic Acids Res. 2004;1:32.

49. Beier S, Thiel T, Münch T, Scholz U, Mascher M. MISA-web: a web server for microsatellite prediction. Bioinformatics. 2017; https://doi.org/10.1093/ bioinformatics/btx198.

50. Mudunuri SB, Nagarajaram HA. IMEx: imperfect microsatellite extractor. Bioinformatics. 2007;23:1181-7.

51. Chen ZD, Manchester SR, Sun HY. Phylogeny and evolution of the Betulaceae as inferred from DNA sequences, morphology, and paleobotany. Am J Bot. 1999. https://doi.org/10.2307/2656981.

52. Yang $X Y$, Wang ZF, Luo WC, Guo XY, Zhang CH, Liu JQ, Ren GP. Plastomes of Betulaceae and phylogenetic implications. J Syst Evol (JSE). 2019. https:// doi.org/10.1111/jse.12479.

53. Hu G, Cheng L, Lan Y, Cao Q, Huang W. The complete chloroplast genome sequence of Corylus chinensis Franch. Conserv Genet Resour. 2016. https:// doi.org/10.1007/s12686-016-0636-6.

54. Hu G, Cheng L, Lan Y, Cao Q, Huang W. The complete chloroplast genome sequence of the endangered Chinese endemic tree Corylus fargesii. Conserv Genet Resour. 2016. https://doi.org/10.1007/s12686-016-0656-2.

55. Yang Y, Zhou T, Duan D, Yang J, Feng L, Zhao G. Comparative analysis of the complete chloroplast genomes of five Quercus species. Front Plant Sci. 2016. https://doi.org/10.3389/fpls.2016.00959.
56. Dong W, Xu C, Li W, Xie X, Lu Y, Liu Y, et al. Phylogenetic resolution in Juglans based on complete chloroplast genomes and nuclear DNA sequences. Front Plant Sci. 2017. https://doi.org/10.3389/fpls.2017.01148.

57. Zong D, Zhou A, Zhang Y, Zou X, Li D, Duan A, He C. Characterization of the complete chloroplast genomes of five Populus species from the western Sichuan plateau, southwest China: comparative and phylogenetic analyses. PeerJ. 2019. https://doi.org/10.7717/peerj.6386.

58. Cheng L, Huang W, Lan Y, Cao Q, Su S, Zhou Z, et al. The complete chloroplast genome sequence of the wild Chinese chestnut (Castanea mollissima). Conserv Genet Resour. 2017. https://doi.org/10.1007/s12686017-0805-2.

59. Raubeson LA, Peery R, Timothy W, Dziubek CC, Fourcade HM, Booreet JL, et al. Comparative chloroplast genomics: analyses including new sequences from the angiosperms Nuphar advena and Ranunculus macranthus. BMC Genomics. 2007. https://doi.org/10.1186/1471-2164-8-174.

60. Lu RS, Li P, Qiu YX. The complete chloroplast genomes of three Cardiocrinum (Liliaceae) species: comparative genomic and phylogenetic analyses. Front Plant Sci. 2017. https://doi.org/10.3389/fpls.2016.02054.

61. Choi KS, Park S. The complete chloroplast genome sequence of Aster spathulifolius (Asteraceae); genomic features and relationship with Asteraceae. Gene. 2015. https://doi.org/10.1016/j.gene.2015.07.020.

62. Williams AV, Boykin LM, Howell KA, Nevill PG, Small I. The complete sequence of the Acacia ligulata chloroplast genome reveals a highly divergent clpP1 gene. PLoS One. 2015. https://doi.org/10.1371/journal. pone.0125768.

63. Qian J, Song JY, Gao HH, Zhu YJ, Xu J, Pang XH, et al. The complete chloroplast genome sequence of the medicinal plant Salvia miltiorrhiza. PLoS One. 2013. https://doi.org/10.1371/journal.pone.0057607.

64. Jiang D, Zhao Z, Zhang T, Zhong W, Liu C, Yuan Q, et al. The chloroplast genome sequence of Scutellaria baicalensis provides insight into intraspecific and interspecific chloroplast genome diversity in Scutellaria. Genes. 2017. https://doi.org/10.3390/genes8090227.

65. Bousquet J, Strauss SH, Li P. Complete congruence between morphological and $r b c L$-based molecular phylogenies in birches and related species (Betulaceae). Mol Biol Evol. 1992;9:1076-88.

66. Kato H, Oginuma K, Gu Z, Hammel B, Tobe H. Phylogenetic relationships of Betulaceae based on matK sequences with particular reference to the position of Ostryopsis. Acta Phytotaxon Geobot. 1998;49:89-97.

67. Hufford L, Moody ML, Soltis DE. A phylogenetic analysis of Hydrangeaceae based on sequences of the plastid gene matK and their combination with rbcL and morphological data. Int J Plant Sci. 2001;162:835-46.

68. Wang N, Thomson M, Bodles WJA, Crawford RMM, Hunt HV, Featherstone AW, et al. Genome sequence of dwarf birch (Betula nana) and cross-species RAD markers. Mol Ecol. 2013;22:3098-111.

69. Bolger AM, Lohse M, Usadel B. Trimmomatic: a flexible trimmer for Illumina sequence data. Bioinformatics. 2014. https://doi.org/10.1093/ bioinformatics/btu170.

70. Greiner S, Lehwark P, Bock R. OrganellarGenomeDRAW (OGDRAW) version 1. 3.1: expanded toolkit for the graphical visualization of organellar genomes. Nucleic Acids Res. 2019. https://doi.org/10.1093/nar/gkz238.

71. Edgar RC. MUSCLE: multiple sequence alignment with high accuracy and high throughput. Nucleic Acids Res. 2004;19:1792-7.

72. Tamura K, Nei M. Estimation of the number of nucleotide substitutions in the control region of mitochondrial DNA in humans and chimpanzees. Mol Biol Evol. 1993;10:512-26.

73. Kumar S, Stecher G, Li M, Knyaz C, Tamura K. MEGA X: molecular evolutionary genetics analysis across computing platforms. Mol Biol Evol. 2018;35:1547-9.

\section{Publisher's Note}

Springer Nature remains neutral with regard to jurisdictional claims in published maps and institutional affiliations. 